\title{
NOTE ON UNBIASED ESTIMABILITY OF THE LARGER OF TWO MEAN VALUES
}

Abstract. An unbiased estimator of the larger of two mean values is constructed provided that the number of observations is random.

1. Introduction. This paper is motivated by the result of Kumar and Sharma (1993). They gave general conditions under which there does not exist an unbiased estimator of the larger of the two components of the expectation of a two-dimensional random vector. They applied this result to show unbiased inestimability of the larger of the two location parameters in the case of uniform as well as double exponential densities.

In Section 2 we give a general scheme for constructing unbiased estimators with a random sample size which can be applied to several situations where there does not exist an unbiased estimator of a given parameter. For example, a somewhat similar idea was investigated earlier by Rychlik (1995) [see also Rychlik (1990)] in order to construct an unbiased estimator of the unknown density of a given random variable.

In Section 3 we consider the problem of unbiased estimability of the larger of two mean values.

2. Unbiased estimation from the sample of a random size. Assume that $T_{1}, T_{2}, \ldots$ is a sequence of $k$-dimensional random vectors with distributions depending on some parameter $\theta \in \Theta$, where $\Theta$ is a subset of $\mathbb{R}^{k}$. The question is how to construct an unbiased estimator of the parameter $\theta$ when it is known that no $T_{i}$ is unbiased. The following proposition characterizes the situation when randomizing the sample size provides an unbiased estimator of $\theta$.

1991 Mathematics Subject Classification: Primary 62F10.

Key words and phrases: unbiased estimation, ordered parameters.

Research partially supported by the NRC-NAS Grant. 
Proposition 2.1. Let $N$ be a random variable with the distribution independent of $\theta$ and such that $P(N=n)=p_{n}, p_{n}>0$ for $n=1,2, \ldots$ and $\sum_{n=1}^{\infty} p_{n}=1$. Then there exists a sequence of statistics $\left\{\theta_{n}^{*}, n=1,2, \ldots\right\}$ independent of $N$ and such that

$$
E \theta_{N}^{*}=\theta, \quad \text { for every } \theta \in \Theta,
$$

if and only if there exists an asymptotically unbiased estimator $T_{n}, n=$ $1,2, \ldots$, of the parameters $\theta \in \Theta$ which is independent of $N$ and such that

$$
\sum_{n=1}^{\infty} E_{\theta}\left|T_{n}-T_{n-1}\right|<\infty
$$

Proof. We first prove the "if" part. Assume that $T_{n}$ is an asymptotically unbiased estimator of $\theta \in \Theta$, i.e.

$$
\lim _{n \rightarrow \infty} E_{\theta} T_{n}=\theta .
$$

Assume that $N$ is a random variable independent of $\left\{T_{1}, T_{2}, \ldots\right\}$. For every $n=1,2, \ldots$, define

where $T_{0} \equiv 0$. Then

$$
\theta_{n}^{*}=\frac{T_{n}-T_{n-1}}{p_{n}},
$$

$$
\begin{aligned}
E_{\theta}\left|\theta_{N}^{*}\right| & =E_{\theta}\left(E_{\theta}\left(\left|\theta_{N}^{*}\right| \mid N\right)\right)=\sum_{n=1}^{\infty} E_{\theta}\left(\left|\frac{T_{N}-T_{N-1}}{p_{N}}\right| \mid N=n\right) p_{n} \\
& =\sum_{n=1}^{\infty} E_{\theta}\left|T_{n}-T_{n-1}\right|<\infty
\end{aligned}
$$

Moreover,

$$
\begin{aligned}
E_{\theta} \theta_{N}^{*} & =E_{\theta}\left(E_{\theta}\left(\theta_{N}^{*} \mid N\right)\right)=\sum_{n=1}^{\infty} E_{\theta}\left(\frac{T_{N}-T_{N-1}}{p_{N}} \mid N=n\right) p_{n} \\
& =\lim _{n \rightarrow \infty} E_{\theta} T_{n}=\theta
\end{aligned}
$$

which proves the "if" part. Now assume that there is a sequence of statistics $\left\{\theta_{n}^{*}, n=1,2, \ldots\right\}$ independent of $N$ and such that

$$
E_{\theta} \theta_{N}^{*}=\theta, \quad \text { for every } \theta \in \Theta .
$$

For every $n=1,2, \ldots$, define

$$
T_{n}=\theta_{1}^{*} p_{1}+\ldots+\theta_{n}^{*} p_{n} .
$$

Since

$$
E_{\theta}\left|\theta_{N}^{*}\right|=\sum_{n=1}^{\infty} E_{\theta}\left|T_{n}-T_{n-1}\right|,
$$


where $T_{0}=0$, the series on the right side converges. Moreover, $\left\{T_{n}, n=\right.$ $1,2, \ldots\}$ is asymptotically unbiased. Indeed,

$$
\lim _{n \rightarrow \infty} E_{\theta} T_{n}=\lim _{n \rightarrow \infty} \sum_{i=1}^{n} p_{i} E_{\theta} \theta_{i}^{*}=E_{\theta}\left(E_{\theta}\left(\theta_{N}^{*} \mid N\right)\right)=\theta .
$$

So in order to construct an unbiased estimator of some parameter it is enough to find an asymptotically unbiased sequence of estimators $\left(T_{n}\right.$, $n=1,2, \ldots)$ for which $\sum_{n=1}^{\infty} E_{\theta}\left|T_{n}-T_{n-1}\right|<\infty$. The next section concerns finding an unbiased estimator of the greater of two expectations.

3. Unbiased estimability of the larger of two expectations. We start with two simple but useful lemmas.

Lemma 3.1. Let $Z_{1}, \ldots, Z_{n}$ be i.i.d. random variables with $E Z_{1}=m$ and $\sigma^{2}=\operatorname{Var} Z_{1}<\infty$. Let $\xi_{n}=n^{-1} \sum_{i=1}^{n} Z_{i}, \xi_{n}^{+}=\max \left(\xi_{n}, 0\right)$ and $\xi_{n}^{-}=$ $\max \left(-\xi_{n}, 0\right)$. Then

$$
\begin{aligned}
\operatorname{Var}\left|\xi_{n}\right| & =\frac{\sigma^{2}}{n}-4 \int \xi_{n}^{-}(\omega) d P(\omega)\left[\int \xi_{n}^{-}(\omega) d P(\omega)+m\right] \\
& =\frac{\sigma^{2}}{n}-4 \int \xi_{n}^{+}(\omega) d P(\omega)\left[\int \xi_{n}^{+}(\omega) d P(\omega)-m\right] .
\end{aligned}
$$

Proof. Observe that $E \xi_{n}^{2}=\sigma^{2} / n+m^{2}$ and $\operatorname{Var}\left|\xi_{n}\right|=E \xi_{n}^{2}-\left(E\left|\xi_{n}\right|\right)^{2}$. Since $E\left|\xi_{n}\right|=E \xi_{n}+2 \int \xi_{n}^{-}(\omega) d P(\omega)$, therefore

$$
\begin{aligned}
\operatorname{Var}\left|\xi_{n}\right| & =\sigma^{2} / n+m^{2}-\left[m+2 \int \xi_{n}^{-}(\omega) d P(\omega)\right]^{2} \\
& =\sigma^{2} / n-4 \int \xi_{n}^{-}(\omega) d P(\omega)\left[m+\int \xi_{n}^{-}(\omega) d P(\omega)\right] .
\end{aligned}
$$

The second equality can be proven in an analogous way.

Remark 3.2. In order to evaluate $\int \xi_{n}^{-} d P$ when $m>0$ (or $\int \xi_{n}^{+} d P$ when $m<0$ ), one could apply first the Cauchy inequality and next the Chebyshev inequality. Indeed, from the Cauchy inequality we get

$$
\left[\int \xi_{n}^{-}(\omega) d P(\omega)\right]^{2} \leq \int\left[\xi_{n}^{-}(\omega)\right]^{2} d P(\omega) P\left(\xi_{n}<0\right) .
$$

Now, observe that

$$
P\left(\xi_{n}<0\right)=P\left(\frac{1}{n} \sum_{i=1}^{n} Z_{i}-m<-m\right) \leq P\left(\left|\frac{1}{n} \sum_{i=1}^{n} Z_{i}-m\right|>m\right) .
$$

Hence, by the Chebyshev inequality, we obtain

$$
P\left(\xi_{n}<0\right) \leq \frac{\sigma^{2}}{n m^{2}} .
$$


And finally,

$$
\int \xi_{n}^{-}(\omega) d P(\omega) \leq\left[\frac{\sigma^{2}}{n}+m^{2}\right]^{1 / 2} \frac{\sigma}{m \sqrt{n}} .
$$

So when $m>0, \int \xi_{n}^{-} d P$ decreases at least as fast as $n^{-1 / 2}$. The lemma below shows that the rate of decrease is in fact $n^{-1}$.

LEMMA 3.3. Under the assumptions of Lemma 3.1,

$$
\begin{aligned}
\int \xi_{n}^{-}(\omega) d P(\omega) & \leq \frac{1}{2} \sqrt{m^{2}+\sigma^{2} / n}-\frac{1}{2} m & & \text { if } m>0 \\
\int \xi_{n}^{+}(\omega) d P(\omega) & \leq \frac{1}{2} \sqrt{m^{2}+\sigma^{2} / n}+\frac{1}{2} m & & \text { if } m<0 .
\end{aligned}
$$

Proof. Suppose that $m>0$. From the equality (1) of Lemma 3.1 it follows that $x \equiv \int \xi_{n}^{-} d P$ satisfies the inequality

$$
\frac{\sigma^{2}}{n}-4 x(m+x) \geq 0 .
$$

From (3) we get the bound

$$
\int \xi_{n}^{-}(\omega) d P(\omega) \leq \frac{1}{2} \sqrt{m^{2}+\sigma^{2} / n}-\frac{1}{2} m .
$$

The case $m<0$ can be treated analogously by applying (2) instead of (1).

Let $X_{1}, X_{2}, \ldots$ be i.i.d. random variables with a common expectation $\theta_{1}$ and variance $\sigma_{1}^{2}$. Let $Y_{1}, Y_{2}, \ldots$ be i.i.d. random variables with a common expectation $\theta_{2}$ and variance $\sigma_{2}^{2}$. Assume that $\left\{X_{i}, i=1,2, \ldots\right\}$ and $\left\{Y_{i}\right.$, $i=1,2, \ldots\}$ are mutually independent. The problem of our interest is to estimate $\vartheta=\max \left(\theta_{1}, \theta_{2}\right)$. Let us define the following estimator of $\vartheta$ :

$$
\widehat{\vartheta}_{n}=\max \left(\frac{1}{n} \sum_{i=1}^{n} X_{i}, \frac{1}{n} \sum_{i=1}^{n} Y_{i}\right) \text {. }
$$

Let $\nu$ denote the vector of parameters $\left(\theta_{1}, \theta_{2}\right)$. The lemma below provides evaluations of the bias and of the variance of $\widehat{\vartheta}_{n}$.

LEMMA 3.4. Under the above assumptions the following inequalities hold:

(i) $0 \leq E_{\nu} \widehat{\vartheta}_{n}-\vartheta \leq \frac{1}{2}\left\{\left[\left(\sigma_{1}^{2}+\sigma_{2}^{2}\right) / n+\left(\theta_{1}-\theta_{2}\right)^{2}\right]^{1 / 2}-\left|\theta_{1}-\theta_{2}\right|\right\}$;

(ii) $\operatorname{Var}_{\nu} \widehat{\vartheta}_{n} \leq\left(\sigma_{1}^{2}+\sigma_{2}^{2}\right) / n$.

Proof. (i) Observe that $\max \left(a_{1}, a_{2}\right)=\frac{1}{2}\left(a_{1}+a_{2}\right)+\frac{1}{2}\left|a_{1}-a_{2}\right|$ for any $a_{1}, a_{2} \in \mathbb{R}$. Hence

$$
E_{\nu} \widehat{\vartheta}_{n}-\vartheta=\frac{1}{2}\left[E_{\nu}\left|\frac{1}{n} \sum_{i=1}^{n}\left(X_{i}-Y_{i}\right)\right|-\left|\theta_{1}-\theta_{2}\right|\right] .
$$

Clearly, (5) implies the lower bound in (i). 
Now we will prove the upper bound. Let $\xi_{n}=n^{-1} \sum_{i=1}^{n}\left(X_{i}-Y_{i}\right)$. Assume for the time being that $m \equiv \theta_{1}-\theta_{2}>0$ and observe that

$$
\begin{aligned}
E_{\nu}\left|\xi_{n}\right| & =\int \xi_{n}^{+}(\omega) d P(\omega)+\int \xi_{n}^{-}(\omega) d P(\omega) \\
& =\theta_{1}-\theta_{2}+2 \int \xi_{n}^{-}(\omega) d P(\omega) .
\end{aligned}
$$

From (5), (6) and Lemma 3.3 the assertion follows. The case $\theta_{1}<\theta_{2}$ can be treated analogously.

(ii) Observe that

$$
\begin{aligned}
\operatorname{Var}_{\nu} \widehat{\vartheta}_{n}= & \frac{1}{4} E_{\nu}\left\{\frac{1}{n} \sum_{i=1}^{n}\left(X_{i}+Y_{i}\right)+\left|\frac{1}{n} \sum_{i=1}^{n}\left(X_{i}-Y_{i}\right)\right|\right. \\
& \left.-\theta_{1}-\theta_{2}-E_{\nu}\left|\frac{1}{n} \sum_{i=1}^{n}\left(X_{i}-Y_{i}\right)\right|\right\}^{2} \\
= & \frac{1}{4}\left\{E_{\nu}\left[\frac{1}{n} \sum_{i=1}^{n}\left(X_{i}+Y_{i}\right)-\theta_{1}-\theta_{2}\right]^{2}+\operatorname{Var}_{\nu}\left|\xi_{n}\right|\right. \\
& \left.+2 E_{\nu}\left[\frac{1}{n} \sum_{i=1}^{n}\left(X_{i}+Y_{i}\right)-\theta_{1}-\theta_{2}\right]\left[\left|\xi_{n}\right|-E_{\nu}\left|\xi_{n}\right|\right]\right\} \\
\leq & \frac{1}{4}\left\{\sqrt{\frac{\sigma_{1}^{2}+\sigma_{2}^{2}}{n}}+\sqrt{\operatorname{Var}_{\nu}\left|\xi_{n}\right|}\right\}^{2},
\end{aligned}
$$

where the last inequality follows from the Cauchy inequality. From Lemma 3.1 it follows that $\operatorname{Var}_{\nu}\left|\xi_{n}\right| \leq\left(\sigma_{1}^{2}+\sigma_{2}^{2}\right) / n$. Applying this to (7), we get the assertion.

From Proposition 2.1 and Lemma 3.4 we get the following result.

Proposition 3.5. Let $N$ be a random variable independent of the sequences $\left(X_{1}, X_{2}, \ldots\right)$ and $\left(Y_{1}, Y_{2}, \ldots\right)$ and such that $P(N=n)=p_{n}, p_{n}>0$ for all $n=1,2, \ldots$ and $\sum_{n=1}^{\infty} p_{n}=1$. Let $k_{n}$ be a sequence of integers such that $k_{n} \nearrow \infty$ as $n \rightarrow \infty$. Define a sequence of statistics $\left\{T_{0}, T_{1}, \ldots\right\}$ by

$$
T_{0}=0 \quad \text { and } \quad T_{n}=\widehat{\vartheta}_{k_{n}} \quad \text { for } n=1,2, \ldots,
$$

where the $\widehat{\vartheta}_{k_{n}}$ are defined by (4).

(i) If $\sum_{n=1}^{\infty} 1 / \sqrt{k_{n}}<\infty$, then $\vartheta_{N}^{*}=\left(T_{N}-T_{N-1}\right) / p_{N}$ is an unbiased estimator of $\vartheta=\max \left(\theta_{1}, \theta_{2}\right)$.

(ii) If , additionally, $\sum_{n=1}^{\infty}\left(k_{n} p_{n}\right)^{-1}<\infty$, then $\vartheta_{N}^{*}$ has a finite variance.

Proof. (i) From Lemma 3.4(i), it follows that $\left\{T_{n}, n=1,2, \ldots\right\}$ is asymptotically unbiased. Since for $n>1$,

$$
E_{\nu}\left|T_{n}-T_{n-1}\right| \leq \sqrt{\operatorname{Var}_{\nu} T_{n}}+\sqrt{\operatorname{Var}_{\nu} T_{n-1}}+E_{\nu} T_{n}-\vartheta+E_{\nu} T_{n-1}-\vartheta
$$


using Lemma 3.4(i), (ii), we get

$$
\begin{aligned}
E_{\nu}\left|T_{n}-T_{n-1}\right| & \leq 2 \sqrt{\frac{\sigma_{1}^{2}+\sigma_{2}^{2}}{k_{n-1}}}+\left[\frac{\sigma_{1}^{2}+\sigma_{2}^{2}}{k_{n-1}}+\left(\theta_{1}-\theta_{2}\right)^{2}\right]^{1 / 2}-\left|\theta_{1}-\theta_{2}\right| \\
& \leq 2 \sqrt{\frac{\sigma_{1}^{2}+\sigma_{2}^{2}}{k_{n-1}}}+\frac{\sigma_{1}^{2}+\sigma_{2}^{2}}{2\left|\theta_{1}-\theta_{2}\right| k_{n-1}} .
\end{aligned}
$$

From the above inequality and the assumption of (i), it follows that

$$
\sum_{n=1}^{\infty} E_{\nu}\left|T_{n}-T_{n-1}\right|<\infty
$$

and, by Proposition 2.1, $\vartheta_{N}^{*}$ is unbiased.

(ii) Notice that

$$
E_{\nu}\left(\vartheta_{N}^{*}\right)^{2}=E_{\nu}\left\{E_{\nu}\left[\frac{T_{N}-T_{N-1}}{p_{N}}\right]^{2} \mid N\right\}=\sum_{n=1}^{\infty} \frac{E_{\nu}\left(T_{n}-T_{n-1}\right)^{2}}{p_{n}} .
$$

From Lemma 3.4, we get

$$
\begin{aligned}
E_{\nu} & \left(T_{n}-T_{n-1}\right)^{2} \\
& =E_{\nu}\left[T_{n}-E_{\nu} T_{n}-\left(T_{n-1}-E_{\nu} T_{n-1}\right)+E_{\nu} T_{n}-E_{\nu} T_{n-1}\right]^{2} \\
& \leq \operatorname{Var}_{\nu} T_{n}+\operatorname{Var}_{\nu} T_{n-1}+\left(E_{\nu} T_{n}-E_{\nu} T_{n-1}\right)^{2}-2 \operatorname{Cov}_{\nu}\left(T_{n}, T_{n-1}\right) \\
& \leq\left(\sqrt{\operatorname{Var}_{\nu} T_{n}}+\sqrt{\operatorname{Var}_{\nu} T_{n-1}}\right)^{2}+\left[E_{\nu}\left(T_{n}-T_{n-1}\right)\right]^{2} \\
& \leq 4\left[\frac{\sigma_{1}^{2}+\sigma_{2}^{2}}{k_{n-1}}+\left(\frac{\sigma_{1}^{2}+\sigma_{2}^{2}}{2 k_{n-1}\left|\theta_{1}-\theta_{2}\right|}\right)^{2}\right] .
\end{aligned}
$$

From (8), (9) and the assumption of (ii), it follows that $E_{\nu}\left(\vartheta_{N}^{*}\right)^{2}<\infty$.

Remark 3.6. One can easily give examples of sequences of integers $k_{n}$ satisfying the assumption of (ii) of Proposition 3.5 and such that $\sum_{n=1}^{\infty} k_{n} p_{n}$ $<\infty$, which means that the average number of observations needed to construct an unbiased estimator of $\vartheta$ is finite. In view of Proposition 3.5(ii) in order to have a finite variance, $\vartheta_{N}^{*}$ needs a random number of observations which does not have a finite expectation. Therefore the following problem arises: does there exist a sequential estimator $\widehat{\vartheta}_{M}$ of $\vartheta=\max \left(\theta_{1}, \theta_{2}\right)$ based on a random number $M$ of observations for which both $E_{\nu} M$ and $\operatorname{Var}_{\nu} \widehat{\vartheta}_{M}$ are finite?

Acknowledgements. I wish to thank Tomek Rychlik very much for providing me with two of his papers and for a helpful discussion. 


\section{References}

S. Kumar and D. Sharma (1993), Unbiased inestimability of the larger of two parameters, Statistics 24, 137-142.

T. Rychlik (1995), A class of unbiased kernel estimators of a probability density function, Appl. Math. (Warsaw) 22, 485-497.

T. Rychlik (1990), Unbiased nonparametric estimation of the derivative of the mean, Statist. Probab. Lett. 10, 329-333.

LESłAW GAJEK

INSTITUTE OF MATHEMATICS

INSTITUTE OF MATHEMATICS

POLISH ACADEMY OF SCIENCES

TECHNICAL UNIVERSITY OF ŁÓDŹ

P.O. BOX 137

AL. POLITECHNIKI 11

00-950 WARSZAWA, POLAND

90-924 ŁÓDŹ, POLAND

Received on 15.12.1994 